\title{
Potential antibacterial activity of crude extracts and silver nanoparticles synthesized from Sargassum wightii
}

\author{
*Vinoth Kumar Thirumalairaj ${ }^{1}$, Mahitha Puthanpurayal Vijayan¹, Geetharamani Durairaj², Lakshmanasenthil Shanmu- \\ gaasokan ${ }^{1}$, Rincy Yesudas', Suja Gunasekaran ${ }^{1}$ \\ ${ }^{1}$ School of Biological Sciences, CMS College of Science and Commerce, Coimbatore-641 049, Tamil Nadu, India \\ ${ }^{2}$ Department of Microbiology, Dr. NGP Arts and Science College, Coimbatore-641048, Tamil Nadu, India
}

\begin{abstract}
The present work investigates the antibacterial activity of silver nanoparticles (Ag-NPs) synthesized by biological method using Sargassum wightii. The fresh live seaweed was collected from the Mandapam coast of Tamilnadu, India. Solvent extract was prepared using acetone, petroleum ether and methanol. Aqueous extract of the seaweed was also used for the synthesis of silver Ag-NPs. Seaweed extract is used as a reducing agent of $2 \mathrm{mM}$ silver nitrate solution for the synthesis of Ag-NPs. Periodical monitoring of reaction mixture was done using UV-vis spectroscopy at $300-750 \mathrm{~nm}$. The scanning electron microscopy (SEM) of the sample confirms the presence of Ag-NPs. The antibacterial activity of solvent extract was done by Minimal inhibitory concentration (MIC) assay. The methanol extract of the seaweed at a concentration of $250 \mu \mathrm{g} / \mathrm{ml}$ exhibited potent antimicrobial activity against the test microorganism. The zone of inhibition ranging from $8-14 \mathrm{~mm}$ was observed with different extracts. The antibacterial activity of the synthesized Ag-NPs against the organism was also done by MIC test. The MIC of Ag-NPs was found to be $130 \mu \mathrm{g} / \mathrm{ml}$ for all pathogenic microorganisms selected for the study. The zone of inhibition against Bacillus cereus, Bacillus anhtracis, Staphylococcus aureus and Vibrio alginoyticus were found to be 10, 8, 10 and $9 \mathrm{~mm}$, respectively. The synthesized Ag-NPs exhibited significant antimicrobial activity against the selected microorganisms than the solvent extract of seaweed.
\end{abstract}

Key Words: Seaweed, NPs, drug, SEM, pathogen, medicine.

\section{INTRODUCTION}

Seaweeds are the important marine resources and have been used as a source of food and medicine (Rajasekar et al., 2014). It produces variety of primary and secondary metabolites. Over 2,400 secondary metabolites have been isolated and many of which have been reported to have excellent biological activities (Faulkner et al., 2002) such as antibacterial, anti-cancer, anti-diabetic, anti-tumor, anti-coagulant and antioxidant (Lakshmanasenthil et al., 2014).

Seaweeds are the important sources of the primary producers and supply considerably to the carbon budget of the marine ecosystem. Over the past several decades seaweeds have generated an enormous amount of interest in the pharmacological industry with enormous medicinal properties. They are rich in antioxidants such as alkaloids, flavonoids, pigments, carotenoids, enzymes and polysaccharides (Heo et al., 2009).

The significant features of nanotechnology are development and synthesis of nanoparticles (NPs). Nanoparticles are fundamental building blocks of nanotechnology. The progress of biologically stimulated experimental process for synthesis of nanoparticles is evolving into an important aspect of nanotechnology. Nanoparticles have been extensively researched for anticancer, drug deliverv, antiviral, anti-cancer and antibacterial activity (Johnston et al., 2010).

In recent years, Ag-NPs have been widely used in various applications because of their well-known

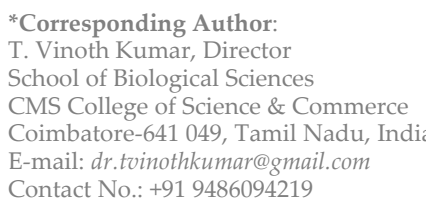

effectiveness in biomedical (Cao et al., 2010), electronic, catalysis and optical applications (Hayward et al., 2000). In particular, the outstanding antimicrobial properties of AgNPs have led to the development of variety of silver nanoparticle products including nanosilver-coated bandages, surgical instruments, contraceptive devices and dental implants (Lohse et al., 2012).

The present study was aimed to evaluate the bioactive compounds and synthesis of nanoparticles from brown seaweed Sargassum wightii against human bacterial pathogens.

\section{MATERIALS AND METHODS}

Collection and identification of seaweed

The fresh live seaweed sample of Sargassum wightii was collected from Mandapam cost of Tamil Nadu, India. The collected seaweed was washed with fresh water to remove epiphytes. Then the sample was brought to laboratory in polythene bags and washed thoroughly with fresh water to remove salt and other extraneous material. The washed sample was shade dried and powdered using an electric mixer. Collected seaweed was identified on the basis of pigmentation, morphology and authenticated (Voucher specimen No: S3/09/2012) by Dr. P. Anantharaman, Associate Professor, CAS in Marine Biology, Chidambaram, Tamil Nadu.

\section{Collection of bacterial culture}

The pathogenic pure cultures of Bacillus cereus, $B$. anthracis, Stapylococcus aureus and Vibrio alginolyticus were obtained from Medical college, Annamalai University, Tamil Nadu. The pure cultures were then sub cultured on nutrient agar slants and preserved under refrigeration for further use. 


\section{Preparation of crude extract of the seaweed}

From the powdered sample, $100 \mathrm{~g}$ was taken and extraction was done by maceration method using three different organic solvents - acetone, petroleum ether and methanol. Dried bio mass was taken in $500 \mathrm{ml}$ conical flasks with $100 \mathrm{ml}$ of solvents and the flasks were continuously stirred in Orbitek ${ }^{\circledR}$ shaker at $200 \mathrm{rpm}$ for 48 hrs. The contents were subjected to centrifugation at 10000 rpm for $30 \mathrm{~min}$. The supernatant was collected and evaporated at room temperature.

\section{Synthesis of Ag-NPs}

The synthesis of Ag-NPs from crude extract was done by taking $10 \mathrm{ml}$ of the sample and mixing with $90 \mathrm{ml}$ of 2 $\mathrm{mM}$ silver nitrate solution $\left(\mathrm{AgNO}_{3}\right)$. The mixture was then stirred using a magnetic stirrer in dark at room temperature for 2 hours. The synthesis of NPs was observed by a change in color of the silver nitrate solution. Periodical UV-visible spectroscopic monitoring of the sample mixture was done at $300-750 \mathrm{~nm}$.

\section{SEM analysis}

The Ag-NPs solution obtained was purified by repeated centrifugation at $5000 \mathrm{rpm}$ for 15 minutes. Ag-NPs so obtained were shade dried at room temperature and powdered. It was submitted for Scanning Electron Microscopic analysis (Hitachi S-4500 SEM machine) at South Indian Textiles Research Association, Coimbatore, Tamil Nadu. The size of the synthesized Ag-NPs from $S$. wightii was characterized using SEM.

\section{Antibacterial activity of crude extract and Ag-NPs}

The antibacterial activity of the seaweed was studied by well diffusion method. Acetone, petroleum ether and methanol extracted compounds of the seaweed was dissolved in DMSO (0.0075 $\mathrm{g}$ in $1 \mathrm{ml}$ of DMSO). The log phase culture of the pathogenic microorganism namely B. anthracis, B. cereus, S. aureus and $V$. alginolyticus was prepared in nutrient broth and adjusted to 0.5 Mcfarlands opacity. Muller Hinton agar was prepared, sterilized and wells of $5 \mathrm{~mm}$ diameter were cut on the agar. The extracts of $S$. wightii was then dispensed into the wells in varying quantity such as $4,9,18$, and $35 \mu$ lo give the final the concentration of $30,61,125$, and $250 \mu \mathrm{g}$ respectively. The plates were then incubated at $37^{\circ} \mathrm{C}$ for $24 \mathrm{hrs}$ in an incubator. The same method was also followed for studying the antibacterial activity of the synthesized AgNPs.

\section{Statistical analysis}

The experiments were conducted in triplicates and the results were expressed as means \pm S.E.M. Statistical analysis were performed with Sigma plot ${ }^{\circledR} 12.5$ software, Systat, USA.

\section{RESULTS}

\section{Identification of seaweed}

The collected seaweed sample was identified as S. wightii on the basis of pigmentation, morphology and authenticated by Dr. P. Anantharaman, Associate Professor, CAS in Marine Biology, Chidambaram.

\section{Silver nanoparticle synthesis}

$\mathrm{Ag}-\mathrm{NPs}$ were synthesized by using the aqueous extract of S. wightii. The formation of nanoparticles was confirmed by visual assessment. The reaction mixture turning to dark brown color from brownish-yellow color indicated the synthesis of Ag-NPs and the excitation of surface

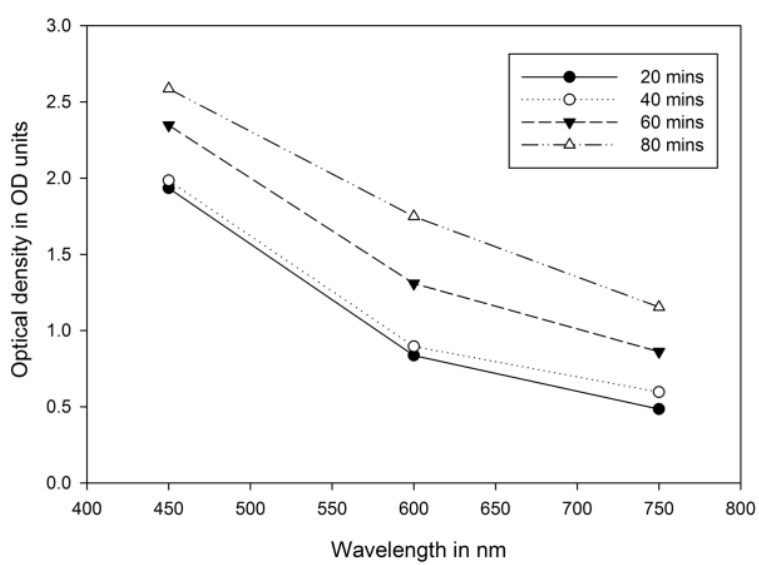

Figure 1: UV-Vis spectra of biologically synthesized silver nanoparticle at different time intervals.

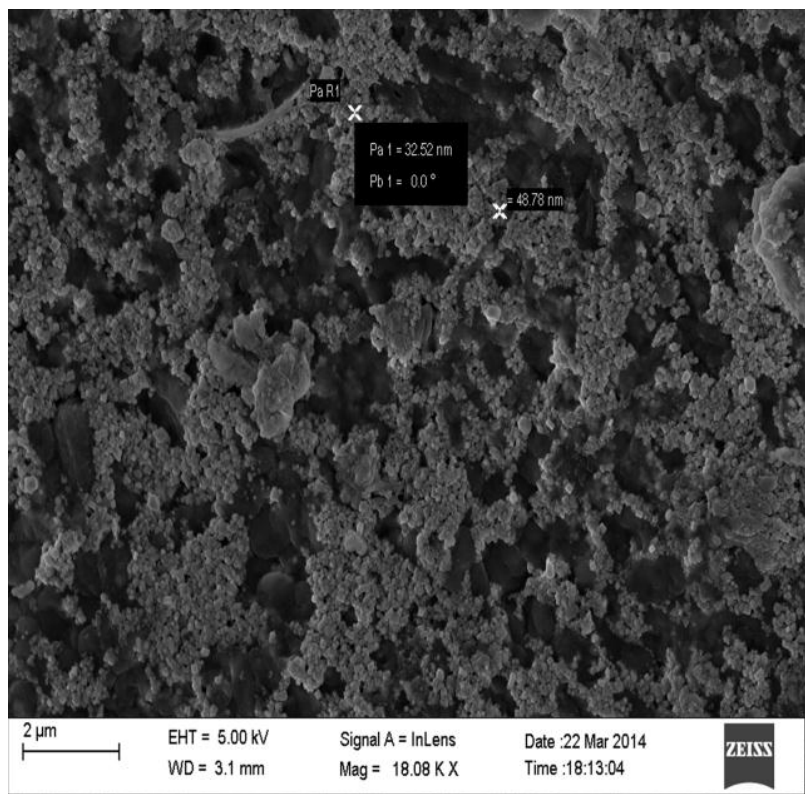

Figure 2: SEM micrograph of silver nanoparticles synthesized by reaction of $2 \mathrm{mM}$ silver nitrate with $S$. wightii.

plasmon resonance effect and reduction of silver nitrate. UV-visible spectrum of reaction mixture at different wave length ranging from $300-800 \mathrm{~nm}$ showed strong absorption peak at $450 \mathrm{~nm}$ indicate the formation of Ag-NPs (figure 1) and this wide peak is due to surface plasmon resonance property of Ag-NPs.

SEM Analysis

The biosynthesized silver nanostructure from $S$. wightii extract was further confirmed by the SEM image. The SEM image shows the high density silver Ag-NPs synthesis. The SEM micrograph of nanoparticle showed that they are spherical shaped and well distributed without aggregation in solution (figure 2) and the average mean size of the Ag-NPs was found to be $48.78 \mathrm{~nm}$.

Antibacterial activity of the solvent extract of $S$. wightii The crude solvent extract (acetone, petroleum ether, and methanol) of seaweed sample were screened for anti- 
Table 1: Antibacterial activity of various solvent extracts on pathogenic microorganisms.

\begin{tabular}{|c|c|c|c|c|c|c|c|c|c|c|c|c|}
\hline \multirow{3}{*}{ Microorganisms } & \multicolumn{12}{|c|}{ Zone of inhibition (mm) } \\
\hline & \multicolumn{4}{|c|}{ Acetone Extract $(\mu \mathrm{g})$} & \multicolumn{4}{|c|}{ Petroleum ether $(\mu \mathrm{g})$} & \multicolumn{4}{|c|}{ Methanol (ug) } \\
\hline & 30 & 61 & 125 & 250 & 30 & 61 & 125 & 250 & 30 & 61 & 125 & 250 \\
\hline B. cereus & - & - & - & $8 \pm 0.03$ & - & - & $9 \pm 0.06$ & $12 \pm 0.02$ & - & $8 \pm 0.02$ & $10 \pm 0.03$ & $12 \pm 0.03$ \\
\hline B. anthracis & - & - & - & $8 \pm 0.02$ & - & $8 \pm 0.07$ & $10 \pm 0.04$ & $13 \pm 0.06$ & - & $9 \pm 0.08$ & $11 \pm 0.05$ & $12 \pm 0.08$ \\
\hline S. aureus & - & - & $9 \pm 0.05$ & $11 \pm 0.07$ & - & - & $8 \pm 0.03$ & $10 \pm 0.03$ & - & $9 \pm 0.07$ & $12 \pm 0.04$ & $14 \pm 0.04$ \\
\hline V. alginolyticus & - & - & $8 \pm 0.03$ & $11 \pm 0.03$ & - & - & $8 \pm 0.05$ & $10 \pm 0.05$ & - & - & - & $9 \pm 0.05$ \\
\hline
\end{tabular}

Table 2: Antibacterial activity of silver nanoparticles.

\begin{tabular}{ccc}
\hline Microorganisams & $\begin{array}{c}\text { Concentration of } \\
\text { Ag-NPs }(\mathbf{1} \boldsymbol{\mu g} / \boldsymbol{\mu l})\end{array}$ & $\begin{array}{c}\text { Diameter of zone of } \\
\text { inhibition (in mm) }\end{array}$ \\
\hline \multirow{3}{*}{ B. cereus } & 52 & $8 \pm 0.04$ \\
& 78 & $11 \pm 0.08$ \\
& 104 & $12 \pm 0.03$ \\
& 130 & $15 \pm 0.09$ \\
\hline B. anthracis & 52 & $9 \pm 0.05$ \\
& 78 & $10 \pm 0.06$ \\
& 104 & $12 \pm 0.03$ \\
& 130 & $13 \pm 0.09$ \\
\hline \multirow{3}{*}{ S. aureus } & 52 & $8 \pm 0.06$ \\
& 78 & $10 \pm 0.05$ \\
& 104 & $13 \pm 0.05$ \\
& 130 & $15 \pm 0.02$ \\
\hline & 52 & $8 \pm 0.09$ \\
& 78 & $9 \pm 0.07$ \\
& 104 & $13 \pm 0.09$ \\
& 130 & $14 \pm 0.04$ \\
\hline
\end{tabular}

bacterial activity against pathogenic organisms namely $B$. cereus, B. anthracis, S. aureus and V. alginolyticus. Acetone extract exhibited the maximum activity against $S$. aureus (13mm at the concentration $250 \mu \mathrm{g}$ ) and moderate activity against $V$. alginolyticus (11mm at the concentration $250 \mu \mathrm{g}$ ) least activity observed against $B$. cereus and $B$. anthracis. The petroleum ether extract of the seaweed showed the highest antibacterial activity against $B$. cereus and $B$. anthracis (12 and $13 \mathrm{~mm}$ respectively) and moderate activity against $S$. aureus and V. alginolyticus $(14 \mathrm{~mm}$ in diameter). The methanolic extract of seaweed showed highest zone against S.aureus (14 $\mathrm{mm}$ for $250 \mu \mathrm{g}$ concentration). It gave moderate activity against $B$. cereus and $B$. anthracis $(12 \mathrm{~mm}$ in diameter for $125 \mu \mathrm{g})$ and minimum zone against $V$. alginolyticus (table 1).

\section{Antibacterial activity of Ag-NPs}

The evaluation of antibiotic resistant pathogenic bacteria has stimulated the search for effective antibacterial agent from various sources. The nanoparticle synthesized from S. wightii was found highly active against the test bacteria. Potent antibacterial activity was observed against $B$. cereus, B. anthracis, $V$. alginolyticus, $S$. aureus at a concentration of $130 \mu \mathrm{g}$ in $130 \mu \mathrm{l}$ (table 2).

The Ag-NPs synthesized from the seaweed at a concentration of $130 \mu \mathrm{g}$ showed very good antibacterial activity against all pathogenic microorganisms subjected for the study. The Diameter of zone of inhibition against B. cereus, B. anthracis, S. aureus and $V$. alginoyticus were found to be $15,13,15$ and $14 \mathrm{~mm}$ respectively.

\section{DISCUSSION}

The silver ions reduction was observed when the silver nitrate was contacted with $S$. wightii extract and it becomes colorless to yellowish brown due to the formation of Ag-NPs. Literature confirms that Ag-NPs can exhibit a size-dependent characteristic surface plasmon resonance which can be deliberated using UV-Vis spectroscopy (Sukdeb et al., 2007).

UV-visible spectrum of reaction mixture showed strong absorption peak at $450 \mathrm{~nm}$ with progressive increase in absorbance upon increasing time until $80 \mathrm{~min}$ as shown in figure 2 . The observed band in this range has been associated with Ag-NPs confirming the synthesis of spherical Ag-NPs with narrow size distribution (Kumar et al., 2012). The microstructure characterization of Ag-NPs was carried out using SEM showed that the Ag-NPs were in narrow size range, with most of the particles with size of 32 and $43 \mathrm{~nm}$.

In this present study, we found that $S$. wightii extract can be a good mediator for the synthesis of Ag-NPs. This green chemistry approach towards the synthesis of AgNPs has many advantages such as ease of scale up, economic viability and independent of use of energy, temperature and toxic chemicals. Applications of such pollution free nanoparticles in medical and electronic applications, makes this method potentially exciting for the large-scale synthesis of other inorganic materials (nanomaterials) (Dubay, 2009).

The results indicate that $S$. wightii can be beneficially used in the nanobiotechnology- based industries for bioinspired synthesis of Ag-NPs. Further studies are underway to characterize the synthesized Ag-NPs.

\section{CONCLUSION}

In this present investigation, environment friendly synthesis of Ag-NPs using macro algae was carried out. This method of Ag-NPs synthesis does not involve any toxic reagents and an alternative for Chemical synthesis methods that lead to presence of some toxic chemical absorbed on the surface that may have adverse effect in the medical applications. Macro algae mediated synthesis is an ecofriendly or nontoxic system and thus has the potential for use in biomedical applications.

\section{ACKNOWLEDGEMENT}

The authors are thankful to the authorities of CMS college of Science and Commerce for their facilities. 


\section{REFERENCES}

Cao XL, Cheng C, Ma YL and Zhao CS (2010) Preparation of silver nanoparticles with antimicrobial activities and the researches of their biocompatibilities. J Mater Sci Mater Med 21: 2861-2868. [DOI]

Dubey M A, Bhadautria S and Kushwah B S (2009) Green synthesis of nanosilver particles from extract of Eucalyptus hybrida (safeda) leaf. Dig. J.Nanomat. Bios 4(3): 537-543. [DOI]

Faulkner DJ (2002) Marine natural products. Nat Products Rep 19: 1-48. [DOI]

Hayward RC, Saville DA and Aksay IA (2002) Electrophoretic assembly of colloidal crystals with optically tunable micropatterns. Nature 404: 56-59

Heo SJ, Hwang JY, Choi JI, Han JS, Kim HJ, Jeon YJ (2009) Diphlorethohydroxycarmalol isolated from Ishige okamurae, a brown algae, a potent alpha-glucosidase and alpha-amylase inhibitor, alleviates postprandia hyperglycemia in diabetic mice. Eur J Pharmacol 615(1-3):252-6. [DOI]

Johnston HJ, Hutchison G, Christensen FM, Peters S, Hankin S, Stone V (April 2010). A review of the in vivo and in vitro toxicity of silver and gold particulates: particle attributes and biological mechanisms responsible for the observed toxicity. Crit. Rev. Toxicol. 40 (4): 32846. [DOI]

Kumar P, Senthamil Selvi S, Lakshmi Prabha A, Prem Kumar K Ganeshkumar RS and Govindaraju M (2012) Synthesis of silver nanoparticles from Sargassum tenerrimum and screening phytochemicals for its antibacterial activity. Nano Biomed Eng 4 (1):12-16. [DOI]
Lakshmanasenthil S, Vinothkumar T, Geetharamani D, Maruthupandi T, Suja G, Sindhu NS (2014) Fucoidan - a noval $\alpha$-amylase inhibitor from Turbinaria ornata with relevance to NIDDM therapy. Biocatal Agricult Biotechnol 3: 67-70. [DOI]

Lohse SE and Murphy CJ (2012) Applications of colloidal inorganic nanoparticles: From medicine to energy. J Am Chem Soc 134:1560715620. [DOI]

Rajasekar T, Priyadharshini P, Kumaran S, Deivasigamani B, Sakthivel M, Balamurugan S, George EGJ (2014) Screening of marine seaweeds for bioactive compound against fish pathogenic bacteria and active fraction analysed by gas chromatography- mass spectrometry. Journal of Coastal Life Medicine 2(5): 367-375. [DOI]

Rashmi C. Vinayak, A. S. Sabu, and Anil Chatterji (2011) Bio-prospecting of a few brown seaweeds for their cytotoxic and antioxidant activities. Evidence-Based Complementary and Alternative Medicine 2011: 9. [DOI]

Sukdeb Pal, Yu Kyung Tak, and Joon Myong Song (2007) Does the Antibacterial Activity of Silver Nanoparticles Depend on the Shape of the Nanoparticle? A Study of the Gram-Negative Bacterium Escherichia coli. Appl Environ Microbiol. 73(6): 1712-1720. [DOI] 